\title{
Respostas fisiológicas agudas do futebol recreacional em mulheres adultas não treinadas
}

\author{
Acute physiological responses of \\ recreational soccer in adult untrained \\ women
}

Rev Bras Ativ Fis Saúde p. 435-444 DOI:

http://dx.doi.org/10.12820/rbafs.v.18n4p435

1 Laboratório de Esforço Físico, Universidade Federal de Santa Catarina, Florianópolis/ SC, Brasil

2Laboratório de Biomecânica, Universidade Federal de Santa Catarina, Florianópolis/ SC, Brasil

3Universidade Estadual de Londrina, Londrina/PR, Brasil

Jaelson Gonçalves Ortiz ${ }^{1,2}$

Fernando Diefenthaeler ${ }^{2}$

Vinícius Milanez ${ }^{3}$

Fabio Yuzo Nakamura

Luiz Guilherme Antonacci Guglielmo ${ }^{1}$

Juliano Fernandes da Silva ${ }^{1}$

\section{Resumo}

Os objetivos deste estudo foram verificar o perfil fisiológico e o padrão de atividade de jogos reduzidos $(7 \mathrm{x} 7$ e $8 \times 8)$ e se os mesmos podem ser utilizados como atividade promotora da aptidão física em mulheres não treinadas. Vinte e três mulheres $(28,9 \pm 3,5$ anos, $165,1 \pm 5,7 \mathrm{~cm}$, $62,1 \pm 8,7 \mathrm{~kg}, 26,6 \pm 5,0 \% \mathrm{G}, 22,8 \pm 2,8 \mathrm{~kg} / \mathrm{m}^{2}$ ) participaram deste estudo. Foram monitorados quatro jogos, sendo dois em cada formato. A frequência cardíaca (FC) foi registrada durante as partidas e amostras de sangue foram coletadas após cada período dos jogos. O perfil de atividade foi mensurado por Global Positioning System (GPS) em uma sessão de cada formato. Os valores percentuais da frequência cardíaca máxima (\%FCmax) durante o primeiro (T1) e o segundo tempo (T2) do jogo nos formatos $7 \times 7$ e $8 \times 8$ foram de $90,2 \pm 4,0 \%$ vs. 89,5 $\pm 4,2 \%$ e de 90,6 $\pm 3,2 \%$ vs. $90,9 \pm 3,6 \%$, respectivamente. Na maior parte do tempo, em ambos os formatos de jogo, as jogadoras permaneceram na intensidade referente ao domínio severo (69\% vs. 74\%, respectivamente). As distâncias totais percorridas em ambos formatos de jogos não apresentaram diferenças significativas $(3363,7 \pm 489,6 \mathrm{~m}$ vs. $3340,6 \pm 487,2 \mathrm{~m}$, para $7 \times 7$ e $8 \mathrm{x} 8$ respectivamente, $p=0,47)$. No entanto, foi observada diferença significativa para distância percorrida entre T1 e T2 do jogo 7x7 (1881,7 $\pm 251,4 \mathrm{~m}$ vs. 1481,9 $\pm 426,0 \mathrm{~m}, \mathrm{p}=0,02)$. Conclui-se que jogos de futebol recreacional realizados nos formatos $7 \mathrm{x} 7$ e $8 \mathrm{x} 8$ podem ser potencialmente utilizados como atividade promotora da aptidão física em mulheres não treinadas.

\section{Palavras-chave}

Esportes coletivos; Aptidão física; Intensidade do exercício.

\begin{abstract}
The aim of this study were to investigate the physiological profile and activity pattern in small-sided games $(7 x 7$ and $8 x 8)$ and whether they can be used as promoter activity of physical fitness in untrained. Twenty-three women $(28.9 \pm 3.5$ years, $165.1 \pm 5.7 \mathrm{~cm}, 62.1 \pm 8.7 \mathrm{~kg}, 26.6 \pm 5.0 \%, 22.8 \pm 2.8 \mathrm{~kg} /$ $\mathrm{m}^{2}$ ) participated in this study. Four matches were monitored, two in each format. Heart rate (HR) was recorded during all matches and blood samples were collected at one match in each format after each period of the game. The activity pattern of the players was measured by Global Positioning System (GPS) in a session of $7 x 7$ and $8 x 8$. The percentages of maximum heart rate (\%HRmax) were $90.2 \pm 4.0 \% \mathrm{vs}$. $89.5 \pm 4.2 \%$, during the first (T1) and second time (T2) of the game in $7 x 7$ format, and $90.6 \pm 3.2 \%$ vs. $90.9 \pm 3.6 \%$ respectively, in the $8 \times 8$ format. Most of the time in both formats of the game, players in the field remained at severe intensity (69\% vs. $74 \%, 7 \times 7$ and $8 \times 8$ formats, respectively). The total distances performed between both matches formats, were not significantly different (3363.7 \pm 489.6 $m$ vs. $3340.6 \pm 487.2 m$, for $7 x 7$ and $8 \times 8$ respectively, $p=0,47)$. However, significant differences were observed for distance between $T 1$ and $T 2$ in the $7 x 7$ game $(1881.7 \pm 251.4 \mathrm{~m}$ vs. $1481.9 \pm 426.0 \mathrm{~m}$, $p=0,02)$. It is concluded from the results obtained that recreational soccer performed in $7 x 7$ and $8 \times 8$ formats can be potentially used to improve physical fitness in untrained women.
\end{abstract}

\section{Keywords}

Team sports; Physical fitness; Exercise intensity. 


\section{INTRODUÇÃO}

O futebol é caracterizado por movimentos intermitentes em ações de baixa, moderada e alta intensidade no qual são executados principalmente corridas com mudanças de direção, chutes e saltos ${ }^{1,2}$. A intensidade média encontrada em jogos de futebol profissional se aproxima do limiar anaeróbio e a distância total percorrida está entre 8 e $13 \mathrm{~km}^{3,4}$. Durante uma partida, jogadores de futebol realizam aproximadamente entre 1000 e 1400 ações de curta duração, sendo que 93\% dos deslocamentos em alta intensidade são executados entre 2 e 19 m,

Uma partida oficial de futebol é disputada com 11 atletas de cada lado (11x11). No entanto, no treinamento têm sido cada vez mais adotados modelos de jogos reduzidos, que variam desde $2 \times 2$ até $8 \times 8^{7,8}$. Esses jogos reduzidos são utilizados em função dos benefícios da sua especificidade, que englobam aspectos técnicos, táticos e físicos ${ }^{3,9}$. A intensidade média durante jogos reduzidos é próxima a $90 \%$ da frequência cardíaca máxima $(\mathrm{FCmax})^{4,10}$. Porém, o número de indivíduos envolvidos e as dimensões do espaço utilizado nesses formatos de jogos tendem a influenciar na intensidade do exercício, aumentando a demanda energética do metabolismo anaeróbio, quando diminui-se o número de atletas envolvidos ${ }^{7,9}$. Por outro lado, em jogos com um número maior de participantes, como $6 \times 6$, as concentrações de lactato tendem a atingir valores próximos à intensidade do limiar anaeróbio ${ }^{1,8,9}$.

Além disso, o jogo de futebol disputado nas suas versões reduzidas $(7 \mathrm{x} 7$ e 8x8, por exemplo) tem se popularizado principalmente pela sua prática recreacional em gramado artificial, pois necessita de um número reduzido de participantes por equipe ${ }^{10,11}$, além de propiciar uma participação maior nas ações com bola em comparação ao futebol tradicional $(11 \times 11)^{1}$.

Recentemente, tem sido investigada a importância do futebol recreacional nas formas reduzidas em indivíduos não atletas e seus benefícios relacionados à saúde e aptidão física ${ }^{12-14}$. A prática regular de futebol recreacional está associada com aumento da potência aeróbia máxima $\left(\mathrm{VO}_{2} \max \right)$, redução da pressão arterial de indivíduos não atletas ${ }^{15,16}$, potenciais aumentos na função neuromuscular e equilíbrio em indivíduos sedentários (jovens e idosos), e redução dos fatores de riscos para fraturas ósseas em mulheres que estão no período pré-menopausa ${ }^{15,17}$.

O diferencial do futebol estaria no fato de ser um modelo com perfil recreativo, realizado em grupo, possibilitando uma prática mais prazerosa, com vantagem sobre um exercício físico tradicional como a corrida, por exemplo ${ }^{11}$. Além disso, a prática regular do futebol parece ser suficiente para promover melhorias relacionadas à saúde, inserindo-se entre os exercícios que atendem às recomendações do American College of Sports Medicine para melhoria da capacidade aeróbia e aptidão física relacionada à saúde, uma vez que proporciona intensidades de $50-85 \%$ do $\mathrm{VO}_{2}$ max ou entre $64-94 \%$ do $\mathrm{FCmax}^{18}$.

Neste contexto, existe um crescente interesse acerca dos benefícios relacionados à prática de futebol recreacional direcionado para a saúde e aptidão física de sujeitos não treinados de diferentes gêneros ${ }^{14,15,17}$. Assim, as respostas fisiológicas agudas e o padrão de atividade do futebol recreacional em mulheres adultas não treinadas ainda precisam ser melhor investigados. Com isso, os objetivos deste estudo foram: (1) examinar e comparar o padrão de atividade de jogos no formato reduzido de 7x7 e 8x8 em mulheres não treinadas e (2) analisar e comparar as respostas de frequência cardíaca $(\mathrm{FC})$ e lactato durante os jogos. 


\section{MÉTODOS}

\section{Sujeitos}

Vinte e três mulheres adultas não treinadas $(28,9 \pm 3,5$ anos, $165,1 \pm 5,7 \mathrm{~cm}, 62,1$ $\left.\pm 8,7 \mathrm{~kg}, 26,6 \pm 5,0 \% \mathrm{G}, 22,8 \pm 2,8 \mathrm{~kg} / \mathrm{m}^{2}\right)$, funcionárias que executavam atividades administrativas de uma empresa elétrica do estado de Santa Catarina participaram deste estudo. Todos os indivíduos foram devidamente informados dos riscos e desconfortos associados com os procedimentos experimentais e a autorização por escrito de todos os participantes foi obtida antes do estudo, depois de uma explicação detalhada da pesquisa. O estudo foi aprovado pelo Comitê de Ética para pesquisas com humanos da Universidade Federal de Santa Catarina - UFSC (P2046, FR429396).

\section{Desenho do Estudo}

As participantes foram monitoradas durante jogos de futebol recreacional no formato $7 \times 7$ e $8 \times 8$. Todas as 23 jogadoras recreacionais neste estudo participaram de ambos os modelos de jogo e tiveram a FC registrada durante as partidas. Quatro jogos no total foram realizados, sendo dois para cada formato. Amostras de sangue foram coletadas em um jogo de cada formato, após cada período de partida $(n=11)$. Neste estudo, o perfil de atividade das jogadoras foi avaliado em uma sessão de 7x7 e uma de $8 \times 8$ ( $\mathrm{n}=10)$, sendo que os mesmos indivíduos participaram de ambos formatos de jogos, na qual usaram a tecnologia Global Positioning System (GPS) (SPI Elite; GPSports Systems, Canberra, Austrália). Durante os jogos, as voluntárias utilizaram dispositivo GPS (massa: $80 \mathrm{~g}$, dimensões: $91 \times 45 \times 21 \mathrm{~mm}$ ), na parte superior traseira do tronco, usando um cinto de neoprene ajustado para as dimensões corporais.

\section{Organização das Sessões de Treinamento}

As sessões foram realizadas em gramado artificial nas dimensões de $55 \mathrm{~m} \mathrm{x} 40 \mathrm{~m}$. A duração dos jogos foi de 50 min (2 tempos de $25 \mathrm{~min}$ ) intercalados com períodos de descanso de $5 \mathrm{~min}$. Os jogos ocorreram em dias separados (72 horas de intervalo). Duas bolas extras estavam disponíveis para evitar grandes retardos no recomeço de jogo. Não foram feitas orientacões táticas durante os jogos por parte dos avaliadores. Os jogos foram realizados no horário entre 17 e 18 horas e a temperatura ambiente esteve entre $20^{\circ}$ e $25^{\circ}$, a umidade relativa do ar esteve entre 65 e $80 \%$. Durante o período de jogos o clima foi seco (sem chuvas) e não apresentou influência significativa de ventos.

\section{Análise do padrão de movimento}

Foram utilizadas as seguintes categorias de locomoção para definir as intensidades: intensidade leve $\left(\leq 2 \mathrm{~km} \cdot \mathrm{h}^{-1}\right)$, intensidade moderada $\left(2 \mathrm{~km} \cdot \mathrm{h}^{-1}\right.$ - abaixo da velocidade de limiar de lactato - vLL), intensidade pesada (entre vLL e velocidade referente ao início do acúmulo do lactato sanguíneo (onset of blood lactate accumulation - vOBLA), intensidade alta (acima do vOBLA até o pico de velocidade PV), intensidade máxima (acima do PV) e intensidade severa (acima do vOBLA, ou seja, intensidade alta+máxima).

\section{Avaliação Antropométrica e Composição Corporal}

A massa corporal (MC) foi medida com aproximação de $100 \mathrm{~g}$ utilizando uma balança digital (TOLEDO, Brasil). A estatura em pé foi medida com resolução de $0,1 \mathrm{~cm}$, utilizando um estadiômetro, que consistia em uma fita métrica fixada à parede em uma 
linha reta em direção ao chão (SANNY, EUA). Estatura e massa corporal foram utilizados para calcular o IMC $\left(\mathrm{kg} / \mathrm{m}^{2}\right)$. Todas as medições antropométricas seguiram os padrões propostos por Petroski ${ }^{19} \mathrm{e}$ foram obtidos por um avaliador treinado, com níveis adequados de erro técnico de medição de acordo com Pederson \& Gore ${ }^{20}$. Para determinar o percentual de gordura corporal $(\% \mathrm{G})$, as dobras cutâneas das regiões: supra-ilíaca, panturrilha e sub-escapular foram determinados (Cescorf, Brasil), sempre no lado direito do voluntário. A densidade corporal estimada (DC) foi realizada de acordo com Petroski ${ }^{19}$, usando a equação de $\operatorname{Siri}^{21}$ (SIRI, 1961) para a conversão de DC em \%G.

\section{Teste Incremental de Laboratório}

Para a determinação do $\mathrm{VO}_{2}$ max, $\mathrm{vVO}_{2} \mathrm{MAX}$, vOBLA, vLL e PV, foi realizado um teste incremental máximo em esteira rolante (Imbramed Millenium Super ATL, 10.200, Brasil). A velocidade inicial foi de $6,0 \mathrm{~km} \cdot \mathrm{h}^{-1}$ (1\% de inclinação), com incrementos de $1,0 \mathrm{~km} \cdot \mathrm{h}^{-1}$ a cada $3 \mathrm{~min}$ com intervalos de $30 \mathrm{~s}$ para coleta de sangue para posterior mensuração do lactato sanguíneo. $O$ teste foi realizado até a exaustão voluntária. Durante o teste, cada sujeito foi verbalmente encorajado a fazer esforço máximo. $\mathrm{O}$ consumo de oxigênio $\left(\mathrm{VO}_{2}\right)$ foi medido a cada respiração usando o sistema Quark CPET calibrado antes de cada teste para assegurar medidas exatas do ar ambiente, do cilindro de gás e turbina, de acordo com as recomendações do fabricante (Cosmed, Rome, Italy). Os dados foram reduzidos para $15 \mathrm{~s} \mathrm{em} \mathrm{média,} \mathrm{o} \mathrm{qual} \mathrm{foi}$ considerado como a menor velocidade na qual o $\mathrm{VO}_{2} \max$ foi encontrado. $\mathrm{O} \mathrm{VO}_{2} \max$ foi definido pelos critérios propostos por Lacour et al. ${ }^{22}$. A FC foi medida durante o teste incremental usando um monitor Polar RS610i (Polar, Kempele, Finland).

\section{Determinação dos Limiares de Transição Fisiológicas}

No final de cada estágio do teste incremental, $25 \mu \mathrm{L}$ de sangue arterial foram coletados do lóbulo da orelha, sem hiperemia para determinar a concentração de lactato. O sangue foi imediatamente transferido para microtubos de polietileno de $1,5 \mathrm{~mL}$ com tampa (tipo Eppendorf), contendo $50 \mu \mathrm{L}$ de solução de fluoreto de sódio a 1\%. As amostras foram analisadas imediatamente após a coleta utilizando um analisador electroquimico (YSI 2700 STAT $^{\circledast}$, Yellow Springs, Ohio, EUA). O limiar de lactato (LL) foi determinado como a intensidade correspondente à concentração fixa de $2,0 \mathrm{mmol} . \mathrm{L}^{-1} \mathrm{e} o$ início do acúmulo sanguíneo de lactato (OBLA) foi determinado por meio de interpolação linear ${ }^{23}$, adotando uma concentração fixa de lactato de $3,5 \mathrm{mmol} . \mathrm{L}^{-1}$.

\section{Domínios de Intensidade}

Foram definidos três domínios de intensidade de esforço com base nos valores dos limiares de transição fisiológicas ${ }^{24}$. A intensidade moderada foi definida como sendo abaixo do LL, a intensidade pesada foi aquela entre o LL e o OBLA, enquanto que a intensidade severa foi definida como acima do OBLA ${ }^{25}$. Para definir o tempo de permanência em cada domínio nos jogos foi adotada a FC correspondente a cada limiar para delimitar os domínios.

\section{Análise estatística}

Foi utilizada a estatística descritiva para apresentação dos resultados (média e desvio-padrão). Foi verificada a normalidade dos dados usando o teste de Shapiro -Wilk. Foi aplicado teste t de Student para amostras dependentes para comparar o primeiro (T1) e o segundo (T2) tempos de jogo, assim como para comparar o modelo $7 \times 7$ com $8 x 8$. ANOVA one way foi utilizada para comparar o tempo de permanência em cada domínio de esforço delimitado pela FC referente aos limia- 
res, durante os jogos, seguido de teste post-hoc de Tukey. $\mathrm{O}$ mesmo procedimento foi utilizado para analisar o padrão de movimento delimitado pelas velocidades pré-estipuladas. O nível de significância adotado foi de 5\% para todas as análises. Todo o tratamento estatístico foi realizado no software SPSS versão 17.0.

\section{RESULTADOS}

Os valores referentes às variáveis cardiorrespiratórias determinados a partir do teste incremental em esteira rolante estão apresentados na Tabela 1. Os resultados referentes ao \%FCmax durante os jogos não foram significativamente diferentes ao se comparar o primeiro (T1) e segundo tempo (T2) de ambos os formatos de jogos (Tabela 2). Do mesmo modo, para as concentrações de lactato sanguíneo, não foram encontradas diferenças significativas entre o T1 e T2 no jogo de formato $7 \mathrm{x} 7$, bem como para $\mathrm{T} 1 \mathrm{e}$ T2 no formato $8 \mathrm{x} 8$ (Tabela 2).

Tabela 1 - Variáveis cardiorrespiratórias determinados a partir do teste incremental em esteira rolante.

\begin{tabular}{lcc}
\hline Variáveis & Média & $\pm \mathrm{DP}$ \\
\hline $\mathrm{VO}_{2} \max \left(\mathrm{ml} \cdot \mathrm{kg}^{-1} \mathrm{~min}^{-1}\right)$ & 38,2 & 6,6 \\
\hline $\mathrm{VVO}{ }_{2} \max \left(\mathrm{Km} \cdot \mathrm{h}^{-1}\right)$ & 10,9 & 1,7 \\
\hline $\mathrm{PV}\left(\mathrm{Km} \cdot \mathrm{h}^{-1}\right)$ & 11,3 & 1,0 \\
\hline $\mathrm{vOBLA}\left(\mathrm{Km} \cdot \mathrm{h}^{-1}\right)$ & 7,3 & 1,1 \\
\hline $\mathrm{vLL}\left(\mathrm{Km} \cdot \mathrm{h}^{-1}\right)$ & 6,2 & 0,8 \\
\hline $\mathrm{FC} \max (\mathrm{bpm})$ & 189,0 & 5,9 \\
\hline $\mathrm{FC}_{\text {OBLA }}(\mathrm{bpm})$ & 166,0 & 8,4 \\
\hline $\mathrm{FC}_{\mathrm{LL}}(\mathrm{bpm})$ & 133,7 & 22,0 \\
\hline
\end{tabular}

$\mathrm{VO}_{2}$ max: Consumo máximo de oxigênio; $\mathrm{VVO}_{2}$ max: mínima velocidade na qual o $\mathrm{VO}_{2}$ max foi alcançado; PV: pico de velocidade; vOBLA: velocidade referente ao início do acúmulo de lactato; vLL: velocidade referente ao limiar de lactato; $\mathrm{FC}_{\text {ОвцA }}$ : freqüência cardíaca encontrada na intensidade referente ao $\mathrm{OBLA} ; \mathrm{FC}_{\mathrm{LL}}$ : freqüência cardíaca encontrada na intensidade referente ao $\mathrm{LL}$.

Tabela 2 - Valores de percentual da frequência cardíaca e lactato sanguíneo durante os jogos 7×7 e 8x8.

\begin{tabular}{ccccccc}
\hline & \multicolumn{2}{c}{$\%$ FCmax $(\%)$} & & \multicolumn{2}{c}{$\begin{array}{c}\text { Concentrações de lactato } \\
\left(\mathrm{mmol} \cdot \mathrm{L}^{-1}\right)\end{array}$} & Média \\
\cline { 1 - 2 } \cline { 5 - 6 } Intensidades & $1^{\circ}$ Tempo & $2^{\circ}$ Tempo & & $1^{\circ}$ Tempo & $2^{\circ}$ Tempo & \\
\hline $7 \times 7$ & $90,2 \pm 4,0$ & $89,5 \pm 4,2$ & $89,9 \pm 4,1$ & $3,5 \pm 1,5$ & $3,7 \pm 1,4$ & $3,6 \pm 1,4$ \\
\hline $8 \times 8$ & $90,6 \pm 3,2$ & $90,9 \pm 3,6$ & $90,7 \pm 3,4$ & $3,7 \pm 1,4$ & $4,2 \pm 1,6$ & $3,9 \pm 1,5$ \\
\hline
\end{tabular}

x= Média; SD = Desvio padrão.

Os valores de FC pico, FC média, distância total e distâncias percorridas em cada intensidade analisada durante os jogos estão descritos na Tabela 3. A distância percorrida durante o T1 foi significativamente maior em relação ao T2 do jogo $7 \times 7(p=0,02)$. No entanto, nenhuma diferença foi encontrada entre os tempos do jogo 8x8 (Tabela 3). Em relação aos valores de FC pico e média, não foram encontradas diferenças significativas entre T1 e T2 nos dois formatos de jogo (Tabela 3). Por outro lado, foi encontrada maior distância percorrida no T1 comparado com o $\mathrm{T} 2$, nas intensidades moderada, pesada, máxima e severa $(\mathrm{p}=0,01 ; \mathrm{p}=0,02 ; \mathrm{p}=0,01$ e $\mathrm{p}=0,03$, respectivamente) com execeção da intensidade leve na qual foi demonstrada maior distância percorrida no T2 em relação ao T1 ( $\mathrm{p}=0,01)$ (Tabela 3).

A Tabela 4 apresenta os percentuais da distância percorrida em cada período de jogo para o tempo dentro das diferentes zonas de intensidade, na qual pode ser 
observada semelhança nos valores relativos ao formato $8 \mathrm{x} 8$ entre os períodos. Em relação ao jogo no formato $7 \times 7$ observou-se diferença significativa $(17,1 \pm 3,7$ vs $21,4 \pm 4,8 \%, \mathrm{p}=0,04)$ para variável baixa intensidade ao se comparar T1 e T2.

A Figura 1 apresenta os resultados do percentual de tempo que a jogadoras permaneceram em cada domínio de intensidade em ambos os formatos de jogo. As participantes permaneceram a maior parte do tempo na intensidade referente ao domínio severo, ou acima da intensidade relativa ao OBLA.

Tabela 3 - Caracterização da frequência cardíaca pico, frequência cardíaca média, distância total e distâncias percorridas em diferentes categorias de locomoção.

\begin{tabular}{|c|c|c|c|c|c|c|}
\hline \multirow[b]{2}{*}{ Intensidades } & \multicolumn{2}{|c|}{ Jogo $7 \times 7$} & \multirow{2}{*}{ Média/Total } & \multicolumn{2}{|c|}{ Jogo $8 \times 8$} & \multirow{2}{*}{ Média/Total } \\
\hline & $1^{\circ} \mathrm{Tempo}$ & $2^{\circ}$ Tempo & & $1^{\circ}$ Tempo & $2^{\circ}$ Tempo & \\
\hline $\mathrm{Fc}_{\text {pico }}(\mathrm{bpm})$ & $191,0 \pm 10,2$ & $188,8 \pm 7,4$ & $189,9 \pm 7,8$ & $190,4 \pm 6,2$ & $187,4 \pm 8,0$ & $188,9 \pm 6,5$ \\
\hline $\mathrm{Fc}_{\text {med }}(\mathrm{bpm})$ & $171,9 \pm 13,7$ & $169,2 \pm 12,0$ & $170,6 \pm 12,3$ & $171,0 \pm 10,3$ & $171,7 \pm 12,7$ & $171,4 \pm 9,8$ \\
\hline Distância Total (m) & $1881,7 \pm 251,4^{*}$ & $1481,9 \pm 426,0$ & $3363,7 \pm 489,6$ & $1699,7 \pm 207,6$ & $1641,0 \pm 437,0$ & $3340,6 \pm 487,2$ \\
\hline Leve $(m)$ & $63,5 \pm 9,1^{*}$ & $82,0 \pm 24,3$ & $145,5 \pm 27,7$ & $74,3 \pm 18,3$ & $71,0 \pm 18,0$ & $145,3 \pm 27,3$ \\
\hline Moderada (m) & $1038,9 \pm 169,8^{*}$ & $908,5 \pm 258,4$ & $1947,3 \pm 388,5$ & $1023,4 \pm 134,1$ & $964,7 \pm 259,3$ & $1988,1 \pm 287,4$ \\
\hline Pesada (m) & $160,06 \pm 88,8^{\#}$ & $112,1 \pm 83,5$ & $272,1 \pm 161,9$ & $138,6 \pm 65,4$ & $134,2 \pm 89,4$ & $272,8 \pm 147,9$ \\
\hline Alta (m) & $435,7 \pm 246,1$ & $271,9 \pm 117,4$ & $707,7 \pm 214,6$ & $336,1 \pm 158,5$ & $322,5 \pm 178,4$ & $658,6 \pm 322,2$ \\
\hline Máxima (m) & $172,5 \pm 95,8^{*}$ & $120,8 \pm 76,9$ & $279,9 \pm 167,5$ & $127,2 \pm 60,1$ & $148,7 \pm 94.3$ & $275,9 \pm 124,0$ \\
\hline Severa $(m)$ & $608,2 \pm 218,4^{\$}$ & $392,7 \pm 163,7$ & $987,6 \pm 199,7$ & $463,3 \pm 139,7$ & $471,2 \pm 198,5$ & $934,5 \pm 287,4$ \\
\hline
\end{tabular}

$\mathrm{x}=$ Média; SD = Desvio padrão; * $-\mathrm{p}=0,01, \#-\mathrm{p}=0,02, \$-p=0,03$ em relação $2^{\circ}$ Tempo.

Tabela 4 - Percentual do tempo nas intensidades em diferentes categorias de locomoção considerando as distâncias percorridas em cada faixa.

\begin{tabular}{|c|c|c|c|c|c|c|}
\hline \multirow[b]{2}{*}{ Intensidades } & \multicolumn{2}{|c|}{ Jogo $7 \times 7$} & \multirow{2}{*}{ \% Tempo Total } & \multicolumn{2}{|c|}{ Jogo $8 \times 8$} & \multirow{2}{*}{ \% Tempo Tota } \\
\hline & $1^{\circ}$ Tempo & $2^{\circ}$ Tempo & & $1^{\circ}$ Tempo & $2^{\circ}$ Tempo & \\
\hline Leve (\%) & $17,1 \pm 3,7^{*}$ & $21,0 \pm 4,8$ & $18,9 \pm 4,6$ & $19,9 \pm 5,8$ & $23,8 \pm 18,3$ & $21,8 \pm 12,0$ \\
\hline Moderada (\%) & $62,2 \pm 7,0$ & $62,7 \pm 4,3$ & $62,4 \pm 5,8$ & $63,6 \pm 6,1$ & $59,9 \pm 14,0$ & $61,8 \pm 10,7$ \\
\hline Pesada (\%) & $5,6 \pm 2,5$ & $4,1 \pm 2,2$ & $4,9 \pm 2,4$ & $5,0 \pm 2,2$ & $4,7 \pm 2,6$ & $4,9 \pm 2,4$ \\
\hline Alta (\%) & $11,6 \pm 6,5$ & $7,8 \pm 2,5$ & $9,7 \pm 4,5$ & $9,0 \pm 4,0$ & $8,6 \pm 4,8$ & $8,8 \pm 4,4$ \\
\hline Máxima (\%) & $3,5 \pm 2,0$ & $2,9 \pm 2,2$ & $3,2 \pm 2,1$ & $2,5 \pm 1,4$ & $2,9 \pm 2,3$ & $2,7 \pm 1,8$ \\
\hline Severa (\%) & $15,1 \pm 5,9$ & $10,7 \pm 3,8$ & $12,9 \pm 4,9$ & $11,5 \pm 3,7$ & $15,5 \pm 6,9$ & $11,5 \pm 5,3$ \\
\hline
\end{tabular}

$\mathrm{x}=$ Média; SD = Desvio padrão; * $-\mathrm{p}=0,04$ em relação $2^{\circ}$ Tempo.

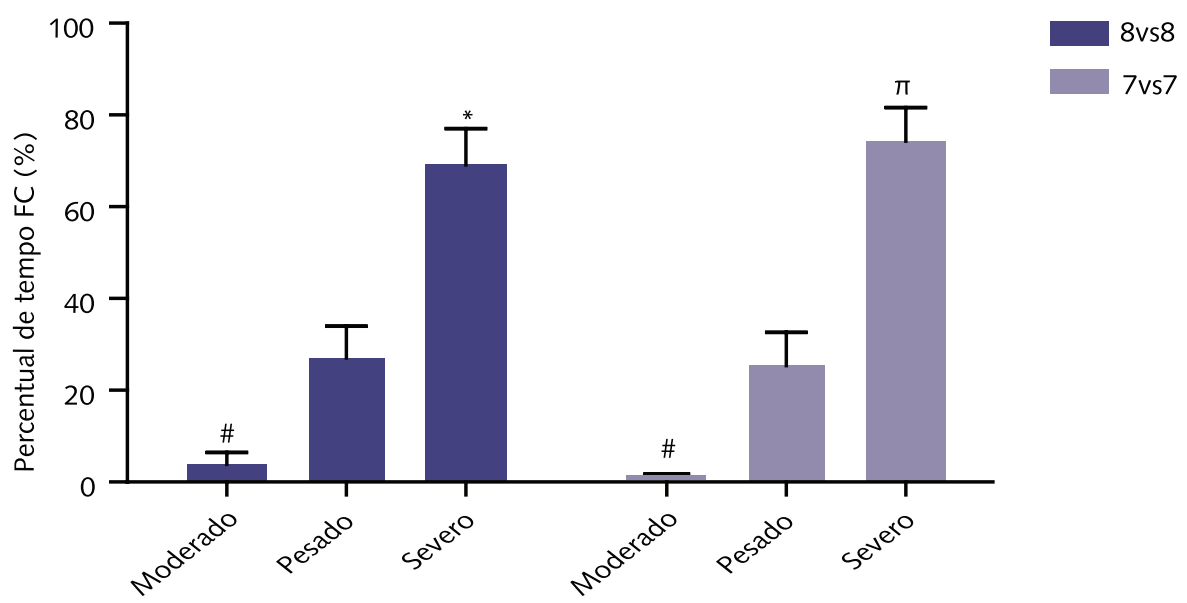

Figura 1 - Percentual de tempo nos domínios de intensidade definidas pela FC associadas aos limiares de transição fisiológica.

${ }^{*} p=0,000$ em relação aos domínios moderado e pesado; $\# p=0,03$ em relação ao domínio pesado; $\pi \mathrm{p}=0,01$ em relação ao domínio pesado. 


\section{DISCUSSÃO}

O objetivo do presente estudo foi verificar as características fisiológicas e o perfil de atividade em jogos de espaço reduzido no formato $7 \times 7$ e $8 \times 8$ em mulheres adultas não treinadas. Os principais achados foram a elevada intensidade dos jogos $(>89 \%$ FCmax) e valores de FC no domínio severo (acima de OBLA) na maior parte da partida ( $70 \%$ do tempo total, em ambos os formatos de jogos), ao passo que o deslocamento ocorreu predominantemente no domínio moderado (entre os limiares).

Brito et al. ${ }^{14}$ utilizaram o formato $5 \times 5$ com dois tempos de $20 \mathrm{~min}$ em homens não treinados e encontraram valores de $\% \mathrm{FCmax}$ mais elevados $(96,9 \pm$ $0,6 \%$ ) que os valores observados no presente estudo (Tabela 2), confirmando que o menor número de jogadores tende a elevar a intensidade dos jogos. Por outro lado, Randers et al. ${ }^{13}$ observaram um \%FCmax de 80,3\% em jogo $7 \mathrm{x} 7$ utilizando quatro tempos de 12 min em grama natural com dimensões de 40 x $60 \mathrm{~m}$, em mulheres não treinadas. As principais diferenças entre os estudos se referem ao tempo realizado e número de períodos de jogo, sendo que o presente estudo foi desenvolvido com dois tempos de $25 \mathrm{~min}$ com $5 \mathrm{~min}$ de intervalo, enquanto que o estudo anterior realizou três períodos de $25 \mathrm{~min}$ com 2 min de recuperação. Os resultados do presente estudo demonstram que a intensidade encontrada em ambos os modelos de jogos ( $7 \mathrm{x} 7$ e 8x8), por meio da FC, está de acordo com as intensidades de exercício recomendadas pelo $A C S M^{19}$ para a promoção da saúde cardiovascular. As intensidades observadas estão próximas ao segundo limiar de transição fisiológica (OBLA) ${ }^{23}$, sugerindo que esta modalidade pode ser adotada como um importante elemento para elevar os níveis de aptidão física em mulheres não treinadas, conforme resultados de estudos prévios ${ }^{11,12,17}$.

As respostas de lactato sanguíneo observadas para o formato de jogo $7 \times 7$ e 8x8 não apresentaram diferenças significativas entre os períodos, assim como entre os formatos. No entanto, ao utilizar um número reduzido de jogadores, $3 \times 3$ em atletas masculinos de futebol, por exemplo, as concentrações de lactato [La], tendem a serem maiores $\left(6,5 \pm 1,5 \mathrm{mmol} \cdot \mathrm{L}^{-1}\right)$, principalmente quando o incentivo verbal é utilizado ${ }^{7}$. Em relação aos valores absolutos de lactato, nossos achados são semelhantes a outros estudos em homens não treinados que demonstraram valores médios de 4,2 \pm 0,6 mmol. $\mathrm{L}^{-1}$ e 4,7 $\pm 0,6 \mathrm{mmol} . \mathrm{L}^{-1}$, para jogo no formato $5 \times 5$ em gramado e areia, respectivamente. ${ }^{14}$ Rampinini et al. ${ }^{7}$, utilizando o formato $6 \times 6$ em três tempos de 4 min em atletas de futebol, observaram valores de $3,4 \pm 1,0 \mathrm{mmol} \cdot \mathrm{L}^{-1} \mathrm{de}$ [La]; porém, os autores não utilizaram incentivo verbal durante o jogo.

Em relação à distância total percorrida durante os jogos, os valores absolutos observados neste estudo foram ligeiramente inferiores aos encontrados no formato $5 \times 5$ (dois tempos de $20 \mathrm{~min}$ ), por Brito et al. ${ }^{14}$ (3726 $\pm 121 \mathrm{~m}$ ). Ao relativizar os resultados acima pela velocidade em metros por minutos, verificamos que o estudo supracitado demonstrou velocidade média de $93,1 \mathrm{~m} \cdot \mathrm{min}^{-1}$, enquanto que no presente estudo as intensidades médias foram de 67,1 e $66,8 \mathrm{~m} \cdot \mathrm{min}^{-1}$ para $7 \mathrm{x} 7$ e 8x8, respectivamente. A maior velocidade média no estudo de Brito et al. ${ }^{14}$ pode ser atribuída ao menor número de participantes por equipe e também devido às diferenças entre os sujeitos de cada estudo, já que a referida pesquisa utilizou homens.

A distância percorrida no formato $8 \times 8$ não apresentou diferença significativa entre os tempos, enquanto que no formato $7 \mathrm{x} 7$ as participantes percorreram uma distância 21\% maior no T1. A partir do perfil de deslocamento (Tabela 1), percebe-se que esta redução no T2 ocorreu simultaneamente ao aumento na distância na categoria de locomoção leve $\left(0-2 \mathrm{~km} \cdot \mathrm{h}^{-1}\right)$. Esse achado está em concordância 
com importantes estudos da literatura que estudaram o jogo na condição 11x11, os quais têm demonstrado maiores distâncias caminhando e maior percentual de tempo parado no segundo tempo ${ }^{27,28}$. A partir dos resultados observados durante o T2 do jogo 7x7 (Tabela 3), sugerimos que as jogadoras do presente estudo adotaram a mesma estratégia, aumentando a distância percorrida em caminhadas e reduzindo a distância em corridas acima da intensidade alta. Outro fator interveniente para redução da distância percorrida pode ter sido o intervalo curto adotado entre os tempos ( $5 \mathrm{~min}$ ), pois enquanto em um jogo oficial o intervalo de $15 \mathrm{~min}$ representa $16 \%$ da duração total, no nosso estudo o intervalo correspondeu a apenas $10 \%$ da duração total.

Um importante achado foi o elevado tempo que as participantes permaneceram com a FC no domínio severo. O maior tempo percentual relativo a $\mathrm{FC}$ encontrado dentro do domínio severo, pode ser explicado pela característica intermitente típica do futebol ${ }^{4-6}$. A ocorrência de momentos de alta intensidade durante as partidas permitiu o aumento e manutenção de elevados valores de $\mathrm{FC}$, mesmo que a movimentação das participantes durante os jogos tenha sido na maior parte realizada em velocidades moderadas ${ }^{2,6}$. Além disso, a baixa aptidão aeróbia das participantes, possivelmente influenciaram o comportamento da $\mathrm{FC}$ em relação ao tempo de permanência no domínio severo ${ }^{24,30}$.

Diversos estudos tem associado um maior tempo de permanência no domínio severo com o aumento na aptidão aeróbia ${ }^{11,29}$. Além disso, o tempo de permanência no domínio severo foi superior ao reportado por Grossl et al. ${ }^{30}(25,6 \pm 19,7 \%)$ em mulheres com perfil aeróbio semelhante, durante aulas de ginástica na modalidade de Power Jump ${ }^{\oplus}$. Do mesmo modo, a média do \%FCmax durante os jogos de futebol recreacional no presente estudo foi superior a $89 \%$, sendo maior que a média do \%FCmax observada $(81,7 \%)$ durante a aula de ciclismo indoor em professores daquela modalidade ${ }^{30}$. Isto reitera que o futebol recreacional em mulheres não treinadas pode ser um importante elemento para melhora da aptidão física, apresentando inclusive intensidade superior a tradicionais modelos utilizados em academias de ginástica.

Portanto, a partir do modelo $7 \times 7$ foi observada maior intensidade na etapa inicial, enquanto que no formato $8 \mathrm{x} 8$ essa variável foi semelhante para ambos os períodos. Essa diferença se deve possivelmente pelas dimensões $(55 \mathrm{~m} \mathrm{x} 40 \mathrm{~m}) \mathrm{e}$ da área de campo correspondente ao espaço utilizado no formato $7 \mathrm{x} 7\left(157 \mathrm{~m}^{2}\right.$ por atletas) e 8x8 (137 $\mathrm{m}^{2}$ por atletas) em relação ao número de jogadoras participantes em cada formato, na qual os praticantes do formato $7 \mathrm{x} 7$ tiveram maior área de campo para percorrer em comparação as jogadoras do jogo no formato 8x8.

Além disso, a alta demanda de esforço observada, principalmente do sistema aeróbio, identificado pela $\mathrm{FC}$, que na maior parte do tempo esteve acima do domínio pesado para ambos os tipos de jogos, sugere a possibilidade de ocorrência de adaptações cardiovasculares a partir da prática de futebol recreacional em modo sistemático e longitudinal em mulheres não treinadas. Os valores de \%FC encontrados para ambos os formatos de jogos no presente estudo estão em concordância com as recomendações do ACSM (2006) para desenvolvimento da aptidão cardiovascular de indivíduos sedentários, pois se encontram próximo à intensidade do OBLA. Portanto, sugere-se novos estudos que possam verificar os efeitos do treinamento a partir de diferentes formatos de jogos de futebol recreacional e seus possíveis benefícios para a aptidão cardiorrespiratória, neuromuscular, assim como, alterações em marcadores bioquímicos relacionados a saúde em indivíduos de diferentes gêneros, idade e nível de condicionamento. 


\section{REFERÊNCIAS}

1. Dellal A, Hill-Haas S, Lago-Penas C, Chamari K. Small-sided games in soccer: amateur vs. professional players: Physiological responses, physical, and technical activities. J Strength Cond Res. 2011; 25: 2371-2381.

2. Iaia FM, Rampinini E, Bangsbo J. High-intensity training in football. Int J Sports Physiol Perform. 2009; 4: 291-306.

3. Reilly T, White C. Small-sided games as an alternative to interval-training for soccer players. J Sports Sci. 2004; 22: 559.

4. Bloomfield J, Polman RCJ, Donoghue PG. Physical demands of different positions in FA Premier League soccer. J Sports Sci Med. 2007 6: 63-70.

5. Stølen W, Chamari K, Castagna C, Wisløff U. Physiology of soccer an update. Sports Med. 2005; 35: 501-536.

6. Vigne G, Gaudino C, Rogowski I, Alloatti G, Hautier C. Activity profile in elite Italian soccer team. Int J Sports Med. 2010; 31 (5): 304-310.

7. Rampinini E, Impellizzeri FM, Castagna C, ABT G, Chamari K, Sassi A, et al. Factors influencing physiological responses to small-sided soccer games. J Sports Sci. 2007; 25 (6): 659-666.

8. Hill-Haas SV, Dawson B, Impellizzeri FM, Coutts A. Physiology of small-sided games training in football. Sports Med. 2011; 3: 199-220.

9. Hill-Haas SV, Coutts AJ, Rowsell GJ, Dawson BT. Generic versus small-sided game training in soccer. Int J Sports Med. 2009; 30: 636-642.

10. Dellal A, Varliette C, Owen A, Chirico E, PIaloux V. Small-sided games versus interval training in amateur soccer players: effects on the aerobic capacity and the ability to perform intermittent exercises with changes of direction. J Strength Cond Res. 2012; 1-9.

11. Krustrup P, Dvorak J, Junge A, Bangsbo J. Executive summary: The health and fitness benefits of regular participation in small-sided football games Scand J Med Sci Sports. 2010; 20 (1): 132-135.

12. Bangsbo J, Nielsen JJ, Mohr M, Randers MB, Krustrup BR, Brito J, et al. Performance enhancements and muscular adaptations of a 16-week recreational football intervention for untrained women. Scand J Med Sci Sports. 2010; 20: 24-30.

13. Randers MB, Nielsen JJ, Krustrup BR, Sundstrup E, Jakobsen MD, Nybo L, et al. Positive performance and health effects of a football training program over 12 weeks can be maintained over a 1-year period with reduced training frequency. Scand J Med Sci Sports. 2010; 20: 80-89.

14. Brito J, Krustrup CP, Rebelo A. The influence of the playing surface on the exercise intensity of small-sided recreational soccer games. Hum Mov Sci. 2012; 31: 946-956.

15. Sundstrup E, Jakobsen MD, Andersen J L, Randers MB, Petersen J, Suetta C, et al. Muscle function and postural balance in lifelong trained male footballers compared with sedentary elderly men and youngsters. Scand J Med Sci Sports. 2010; 20: 90-97.

16. Andersen LJ, Randers MB, Westh K, Martone D, Hansen PR, Junge A, et al. Football as a treatment for hypertension in untrained 30-55-year-old men: a prospective randomized study. Scand J Med Sci Sports. 2010; 20 (1): 98-102.

17. Helge EW, Aagaard P, Jakobsen MD, Sundstrup E, Rander SMB, Karlsson MK, et al. Recreational football training decreases risk factors for bone fractures in untrained premenopausal women. Scand J Med Sci Sports. 2010; 20 (1): 31-39.

18. American College of Sports Medicine. Guidelines for exercise testing and prescription. 7 ed. p.130-173, 2006.

19. Petroski EL, Pires-Neto CS. Validação de equações antropométricas para a estimativa da densidade corporal em mulheres. Rev Bras Ativ Fís e Saúde 1995;1:65-73.

20. Pederson D, Gore C. Erro em medição antropométrica. In: Norton K, Olds T, (eds). Anthropométrica: um livro sobre medidas corporais para o esporte e cursos da área da saúde. Porto Alegre: Artimed Editora. 2005. p. 39-87

21. Siri WE. Body composition from fluid spaces and density. In: Brozek J, Henschel A, (eds). Techniques for measuring body composition. Washington DC: National Academy of Science; 1961. p. 223-44.

22. Lacour JR, Padilla-Magunacelaya S, Chatard JC, Arsac L, Bathelemy, JC. Assessment of running velocity at maximal oxygen uptake. Eur J Appl Physiol. 1991; 62 (2): 77-82. 
23. Berg A, Jokob M, Lehmann HH, Dickhuth G, Huber J. Actualle Aspekte der modern ergometric. Pneumologie. 1990, 44: 2-13.

24. Gaesser GA, Poole DC. The slow component of oxygen uptake kinetics in humans. Exerc Sport Sci Rev. 1996, 24: 35-71.

25. Denadai BS, Gomide EB, Greco CC. The relationship between onset of blood lactate accumulation, critical velocity and maximal lactate steady state in soccer players. J Strength Cond Res. 2005; 19 (2): 364-8.

26. Jones S, Drust B. Physiological and technical demands of $4 \mathrm{v} 4$ and $8 \mathrm{v} 8$ games in elite youth soccer players. Kinesiology. 2007; 39 (2); 150-156.

27. Di Salvo V, Baron R, Tschan H, Montero CFJ, Bachl N, Pigozzi F. Performance characteristics according to playing position in elite soccer. Int J Sports Med. 2007, 28: 222-227.

28. Little T, Williams AG. Measures of exercise intensity during soccer training drills with professional soccer players. J Strength Cond Res. 2007; 21: 367-371.

29. Helgerud J, Rodas G, Kemi OJ, Hoff J. Strength and endurance in elite football players. Int J Sports Med. 2011, 32 (9): 677-82.

30. Gross1 T, Guglielmo LGA, Fernandes da Silva J, Vieira G. Respostas cardiorrespiratórias e metabólicas na aula de ciclismo indoor. Motriz. 2009, 15: 330-339.

Endereço para Correspondência
Fernando Diefenthaeler
Universidade Federal de Santa Catarina
Campus Universitário Reitor João David
Ferreira Lima, Trindade
Florianópolis/SC, CEP: 88040-900;
Fone: (48) 37219924
E-mail: fernando.diefenthaeler@ufsc.br

Recebido 28/08/2013

Revisado 06/09/2013

Aprovado 26/09/2013 